\title{
Quantum phase transitions beyond the Landau-Ginzburg paradigm and supersymmetry
}

\author{
Subir Sachdev*, Xi Yin \\ Department of Physics, Harvard University, Cambridge, MA 02138, United States
}

\section{A R T I C L E I N F O}

\section{Article history:}

Received 6 August 2008

Accepted 4 August 2009

Available online 11 August 2009

\section{Keywords:}

Duality

Supersymmetry

Quantum phase transition

Monopole

Antiferromagnet

Valence bond solid

Deconfinement

\begin{abstract}
A B S T R A C T
We make connections between studies in the condensed matter literature on quantum phase transitions in square lattice antiferromagnets, and results in the particle theory literature on abelian supersymmetric gauge theories in $2+1$ dimensions. In particular, we point out that supersymmetric $U(1)$ gauge theories (with particle content similar, but not identical, to those of theories of doped antiferromagnets) provide rigorous examples of quantum phase transitions which do not obey the Landau-Ginzburg-Wilson paradigm (often referred to as transitions realizing "deconfined criticality"). We also make connections between supersymmetric mirror symmetries and condensed matter particle-vortex dualities.
\end{abstract}

(c) 2009 Elsevier Inc. All rights reserved.

\section{Introduction}

The condensed matter literature has seen much discussion [1-4,6-9,5] on quantum phase transitions that violate the Landau-Ginzburg-Wilson (LGW) paradigm. In such transitions, conventional phases with distinct broken symmetries are generically separated by a second-order quantum critical point e.g. a theory with a global symmetry group $G_{1} \times G_{2}$ has a transition from a phase where $G_{1}$ is broken while $G_{2}$ is preserved, to a phase where $G_{1}$ is preserved and $G_{2}$ is broken. The theory for the critical point is usually not expressed in terms of order parameters which measure $G_{1}$ or $G_{2}$ symmetry breaking, but in terms of 'fractionalized' degrees of freedom, and hence the terminology 'deconfined'.

Non-LGW transitions have been known in $1+1$ dimensions for some time [10], and our interest in this paper is exclusively in $2+1$ dimensions. Examples have been established [11-13,1,2] for $S U\left(N_{f}\right)$ square lattice antiferromagnets for large, but finite, $N_{f}$, as we shall review below. The situation for the physically most interesting case of $N_{f}=2$ remains unsettled: while there is convincing evidence

\footnotetext{
* Corresponding author.

E-mail address: subir_sachdev@harvard.edu (S. Sachdev).
} 
for the emergent degrees of freedom of the deconfined field theory, there are open questions on the nature of the critical point, with some results favoring a first-order transition [4-9].

Although this does not appear to have been recognized in the condensed matter literature, examples of non-LGW transitions have also appeared in the particle theory literature: they are present in supersymmetric field theories in $2+1$ dimensions studied in the early work of Seiberg and collaborators [14-16]. The purpose of our paper is to review the condensed matter and particle theory results in a unified manner. Our aim is to make our discussion intelligible across the boundaries of these fields. We hope to convince the reader that there is a remarkably close analogy between supersymmetric 'deconfined criticality' and the models arising in the study of quantum antiferromagnets. A close connection will also be drawn between the 'mirror symmetry' of the supersymmetric field theories and particle-vortex duality arguments.

An important field theory arising [13,1,2] in the study of the loss of Néel order in $S U\left(N_{f}\right)$ antiferromagnets on the square lattice is the Abelian Higgs model with $N_{f}$ complex scalar fields, $q_{i}, i=1 \ldots N_{f}$, with the Euclidean Lagrangian

$$
\mathcal{L}_{H}\left(q_{i}, A_{\mu}\right)=\left|\left(\partial_{\mu}-i A_{\mu}\right) q_{i}\right|^{2}+r\left|q_{i}\right|^{2}+u\left(\left|q_{i}\right|^{2}\right)^{2}+\frac{1}{g^{2}} F_{\mu v}^{2}
$$

where $A_{\mu}$ is a $U(1)$ gauge field, and $F_{\mu \nu}=\partial_{\mu} A_{v}-\partial_{v} A_{\mu}$ is the 'electromagnetic' field. Here, and henceforth, all Lagrangians are assumed to be integrated over $2+1$ dimensional spacetime to obtain the associated actions. For some purposes, it is useful consider a 'strong-coupling' limit of $\mathcal{L}_{H}$ in which the quartic potential is replaced by a hard constraint $\sum_{i}\left|q_{i}\right|^{2}=1$ : in this case $\mathcal{L}_{H}$ describes the so-called $N C C P^{N_{f}-1}$ model [9] (for non-compact $U(1)$ gauge field $C P^{N_{f}-1}$ model). The universal properties of these two cases are expected to be identical.

For $r$ sufficiently negative, $\mathcal{L}_{H}$ is in the Higgs phase, and the global $S U\left(N_{f}\right)$ symmetry is broken because of the condensation of the $q_{i}$ (for $N_{f}>1$ ). The gauge-invariant 'meson' operators $q_{i}^{*} q_{j}$ are the order parameters for this symmetry breaking: these constitute the Néel order parameters of the $S U\left(N_{f}\right)$ antiferromagnet.

For $r$ sufficiently positive, $S U\left(N_{f}\right)$ symmetry is restored, and $q_{i}$ are massive scalar particles which interact by exchanging $A_{\mu}$ photons. The electric potential between these scalars has the 'Coulomb' form of $\ln (r)$, and so this is referred to as the Coulomb phase.

There is a great deal of interest on the nature of the transition between the Higgs and Coulomb phases as a function of increasing $r$. For $N_{f}=1$ the transition can be either first or second-order depending upon parameters; for large $N_{f}$ it is second-order; and for $N_{f}=2$ the question is the focus of the recent debate [4-9].

Crucial to our purposes here is the fact that $\mathcal{L}_{H}$ enjoys an additional global symmetry, distinct from the $S U\left(N_{f}\right)$ flavor symmetry. This global symmetry is special to $2+1$ dimensions, and is linked to the presence of the 'topological' current

$$
\widetilde{J}_{\mu}=\frac{1}{4 \pi} \epsilon_{\mu \nu \lambda} F_{v \lambda}
$$

which is conserved, $\partial_{\mu} \widetilde{J}_{\mu}=0$, reflecting the conservation of total magnetic flux. We can associate this conservation law with a dual $\widetilde{U}(1)$ global symmetry, and consequently a field operator which changes the magnetic flux will carry $\widetilde{U}(1)$ charge. Because of the presence of the fields $q_{i}$ with unit electrical charge, the Dirac quantization condition implies that the magnetic flux can only change in integer multiples of $2 \pi$. We can therefore introduce the elementary monopole creation operator $\hat{q}$ which carries unit $\widetilde{U}(1)$ charge; these are 'topological disorder operators' for the $U(1)$ gauge theory $[17,19,18,20]$. As written, the continuum theory $\mathcal{L}_{H}$ does not allow for the creation of any monopoles, and this is equivalent to the existence of the global $\widetilde{U}(1)$ symmetry. We can now ask for the fate of this $\widetilde{U}(1)$ symmetry by examining the two-point monopole correlator in both phases. It is not difficult to show that as $|\mathbf{r}| \rightarrow \infty$

$$
\left\langle\hat{q}(\mathbf{r}) \hat{q}^{\dagger}(0)\right\rangle \sim \begin{cases}\exp (-m|\mathbf{r}|), & \text { Higgs phase } \\ \text { const, } & \text { Coulomb phase }\end{cases}
$$


where $m$ is an energy scale characterizing the Higgs phase, of order the Higgs mass. From this it is clear that there are long-range correlations in monopole operator in the Coulomb phase, and consequently the $\widetilde{U}(1)$ symmetry is broken.

To summarize, the Abelian Higgs model has a global $S U\left(N_{f}\right) \times \widetilde{U}(1)$ symmetry. In the Higgs phase, the $S U\left(N_{f}\right)$ symmetry is broken and the $\widetilde{U}(1)$ symmetry is preserved, while in the Coulomb phase the $S U\left(N_{f}\right)$ symmetry is preserved and the $\widetilde{U}(1)$ symmetry is broken.

These conclusions appear to satisfy the requirements of a non-LGW transition, as defined above. However, an objection might be raised that the $\widetilde{U}(1)$ symmetry is 'topological', involves highly nonlocal transformations of the field operators in $\mathcal{L}_{H}$, and so is not directly observable. A remarkable fact of the mapping between $\mathcal{L}_{H}$ and the $S U\left(N_{f}\right)$ quantum antiferromagnets is that the non-local monopole operator can be related to simple local observables expressed in terms of the underlying lattice $S U\left(N_{f}\right)$ spins [13,2]. In particular, for a class of $S U\left(N_{f}\right)$ antiferromagnets (with fundamental matter on the square lattice sites), $\hat{q}$ is proportional to the 'valence bond solid' (VBS) operator. Further, the 'hidden' $\widetilde{U}(1)$ symmetry is not hidden at all, but an enlargement of the spatial $Z_{4}$ rotation symmetry of the square lattice. These connections have been reviewed in other recent articles [21,22], and so we will not describe them further here. All we need for our purposes is the conclusion that the $\widetilde{U}(1)$ symmetry is physical and experimentally measureable, and so the Abelian Higgs model does indeed satisfy the conditions for a non-LGW transition.

For completeness, another significant feature of the connection between $\mathcal{L}_{H}$ and quantum antiferromagnets should be mentioned. While the theory $\mathcal{L}_{H}$ does not permit any monopoles in the strict continuum limit, the fate of the monopoles can only be correctly addressed by the actual short distance physics, which is that of the lattice antiferromagnet. Here it is found that Berry phases [23] lead to large short-distance cancellations between monopoles, and so an additive contribution to the Lagrangian, $\mathcal{L}_{m} \sim \hat{q}+\hat{q}^{\dagger}$ does not appear in the continuum theory [13]. By a careful symmetry analysis of the Berry phases, it was shown [13] that the simplest allowed monopole term was $\mathcal{L}_{m} \sim \hat{q}^{4}+\hat{q}^{\dagger 4}$. Recalling that the $\hat{q}$ carries $\widetilde{U}(1)$ charge, we see that the actual magnetic symmetry of the full theory $\mathcal{L}_{H}+\mathcal{L}_{m}$ is not $\widetilde{U}(1)$, but $Z_{4}$ (with is identified with the square lattice rotation symmetry). It is this $Z_{4}$ symmetry which is broken in the 'Coulomb' phase. However, it has been argued that such a $\hat{q}^{4}$ term is likely irrelevant near the critical point [1,2], and this is supported by numerical studies [4,6,21]. So $\mathcal{L}_{m}$ can be neglected in the immediate vicinity of the critical point, and we will not consider it further here.

The remainder of this paper will describe the analogy between the properties of the above Abelian Higgs model with $N_{f}$ scalars, and the corresponding model with $\mathcal{N}=4$ supersymmetry. Briefly, we simply promote the fields of $\mathcal{L}_{H}$ to the corresponding $\mathcal{N}=4$ multiplets: we promote the scalars $q_{i}$ to hypermultiplets $\mathcal{Q}_{i}$, and the $U(1)$ gauge field $A_{\mu}$ to a $U(1)$ vector multiplet $\mathcal{V}$. With $\mathcal{N}=4$ supersymmetry, the resulting theory is unique and has only a single dimensionful gauge coupling constant $g$; a complete and explicit form of the Lagrangian appears in Section 5. We will explore its phase diagram (i.e. moduli space) and find remarkable analogies with the non-supersymmetric $\mathcal{L}_{H}$.

This generalization to supersymmetric models necessarily involves introduction of Dirac Fermions, both in the matter and gauge multiplets. While precisely this field content is not known to be present in any models of interest in condensed matter, recent work [24-26] has considered a theory in which Dirac Fermions, $\psi_{j}\left(j=1 \ldots N_{d}=4\right)$ with a $U(1)$ charge are added to $\mathcal{L}_{H}$ to obtain

$$
\mathcal{L}_{H \psi}\left(q_{i}, \psi_{j}, A_{\mu}\right)=\left|\left(\partial_{\mu}-i A_{\mu}\right) q_{i}\right|^{2}+r\left|q_{i}\right|^{2}+u\left(\left|q_{i}\right|^{2}\right)^{2}+\frac{1}{g^{2}} F_{\mu \nu}^{2}+\bar{\psi}_{j} \sigma^{\mu}\left(\partial_{\mu}-i A_{\mu}\right) \psi_{j}
$$

where $\sigma^{\mu}$ are the Pauli matrices; these Dirac Fermions represent the Bogoliubov quasiparticle excitations of a $d$-wave superconductor. Note the number of scalars $\left(N_{f}\right)$ and Fermions $\left(N_{d}\right)$ are not equal in the physical case, although this will be the case in the supersymmetric models below. The model $\mathcal{L}_{H \psi}$ is also expected to exhibit a non-LGW transition, which will then be even closer to the supersymmetric models.

We will begin in Section 2 by a review of the 'particle-vortex' or 'Dasgupta-Halperin' duality $[27,28]$ of the Abelian Higgs model with $N_{f}=1$. Then, in Section 3 we will consider the corresponding model with $\mathcal{N}=4$ supersymmetry and $N_{f}=1$. We will show that the formulation of mirror symmetry for this model by Kapustin and Strassler [16] is precisely the same as the exact statement of Dasgupta- 
Halperin duality in Section 2. The analogy between these models also extends to the $N_{f}>1$ case, and this is discussed in Section 4. The subsequent sections will explore the structure of the phases of the $\mathcal{N}=4$ theory.

\section{Abelian Higgs model for $N_{f}=1$}

Here we will present the exact statement of duality properties of the Abelian Higgs model. We consider only the case $N_{f}=1$, with comments about $N_{f}>1$ in Section 4 below.

We write the generating function for the fluxes of the Abelian Higgs model as

$$
Z_{H}\left[\widehat{A}_{\mu}\right]=\int \mathcal{D} q \mathcal{D} A_{\mu} \exp \left(-\int\left[\mathcal{L}_{H}\left(q, A_{\mu}\right)+\frac{1}{2 \pi} \epsilon_{\mu \nu \lambda} \hat{A}_{\mu} \partial_{v} A_{\lambda}\right]\right) .
$$

In general, the functional $Z_{H}\left[\widehat{A}_{\mu}\right]$ will depend upon all the couplings $r, u$, and $g$, and also on the ultraviolet cutoff. However, in the vicinity of a second-order Higgs-Coulomb transition, the functional is dominated by momenta much smaller than the cutoff, and depends only upon universal correlations of the conformal field theory (CFT) describing this transition. The renormalization group fixed point describing the CFT is expected to have only one relevant perturbation, whose strength is characterized by a single mass scale $m$ (which we have to define separately in the Higgs and Coulomb phases). In the scaling limit of the fixed point, the functional $Z_{H}\left[\widehat{A}_{\mu}\right]$ is a universal dimensionless functional of $\widehat{A}_{\mu}$, its momenta $p_{\mu}$, and the mass $m$, all of which have unit scaling dimension. There are no known exact results for this functional, but approximate results can be obtained using the $(4-D)$ expansion ( $D$ is the dimensionality of spacetime), the $1 / N_{f}$ expansion, and by numerical simulations.

In the particle-vortex duality, the flux lines of $\mathcal{L}_{H}$ are mapped onto the world lines of dual particles ('vortices') which are created by the monopole operator $\hat{q}$. Dasgupta and Halperin argued that these dual particles only have short-range interactions, and so are described by the XY model - in the continuum limit, this is the theory $\mathcal{L}_{H}$, but with the no $A_{\mu}$ fluctuations. The generating functional of the dual particle currents in such a theory is given by

$$
Z_{X Y}\left[\widehat{A}_{\mu}\right]=\int \mathcal{D} \hat{q} \exp \left(-\int \mathcal{L}_{H}\left(\hat{q}, \widehat{A}_{\mu}\right)\right)
$$

Now we implicitly assume that the action $\mathcal{L}_{H}$ has different couplings $\hat{r}, \hat{u}$, and $\hat{g}$. The XY model is known to have a second-order critical point, and just as for Eq. (2.1), the functional $Z_{X Y}\left[\widehat{A}_{\mu}\right]$ becomes completely universal in the scaling limit near the fixed point, and depends only upon a single mass scale $\hat{m}$ measuring the deviation from the critical point.

We will now connect the generating functionals in Eqs. (2.1) and (2.2). In general, this requires us to map the couplings $r, u$ and $g$ to $\hat{r}, \hat{u}$, and $\hat{g}$. The form of this mapping is not known, and indeed, depends upon the specific ultraviolet cutoff. However, the central hypothesis of Dasgupta and Halperin [28] was that the RG fixed points describing the transitions in the $N_{f}=1$ Abelian Higgs model and the $\mathrm{XY}$ model are the same. Thus, in the scaling limit near this fixed point, to map the two theories to each other, we need only connect the mass scales $m$ and $\hat{m}$. The latter is easily done, by identifying these with the corresponding particles/vortices in the spectra. After this identification, we then have the remarkable exact duality statement

$$
Z_{H}\left[\widehat{A}_{\mu}\right]=Z_{X Y}\left[\widehat{A}_{\mu}\right] \text {. }
$$

This is one of the very few exact statements about non-supersymmetric CFTs in $2+1$ dimensions; it has not been rigorously established, but the arguments based upon dualities of lattice models appear quite robust.

\section{Abelian gauge theory for $N_{f}=1$ with $\mathcal{N}=4$ supersymmetry}

The statements in Section 2 have a remarkably precise analog in $U(1)$ gauge theories with $\mathcal{N}=4$ supersymmetry, as stated by Kapustin and Strassler [16] (hereafter referred to as KS). 
As we discussed in Section 1, we generalize the scalar $q$ to a $\mathcal{N}=4$ hypermultiplet $\mathcal{Q}$, and the gauge field $A_{\mu}$ to a $\mathcal{N}=4$ vector multiplet $\mathcal{V}$. The precise field content of these hypermultiplets will be specified later in Section 5.

Now we consider the $\mathcal{N}=4$ theory with one hypermultiplet $\mathcal{Q}$ and one vector multiplet $\mathcal{V}$. This theory has a unique Lagrangian, $\mathcal{L}_{S}$, with only one dimensionful gauge coupling constant $g$. This Lagrangian is the analog of Eq. (1.1) and its explicit form of this will appear in Section 5. Next we define a generating functional for the 'fluxes' of $\mathcal{V}$ which is the analog of Eq. (2.1) (as in Eq. (11) of KS):

$$
Z_{\mathrm{SQED}-1}[\widehat{\mathcal{V}}]=\int \mathcal{D V D} \mathcal{Q} \exp \left(-\int\left[\mathcal{L}_{S}(\mathcal{Q}, \mathcal{V})+\mathcal{L}_{B F}(\widehat{\mathcal{V}}, \mathcal{V})\right]\right)
$$

where $\mathcal{L}_{B F}$ is the analog of the Chern-Simons coupling in Eq. (2.1) between two vector fields (see Eq. (8) of KS), and we have dropped the gauge-fixing term included by KS. An advantage of the supersymmetric theory is that it is easy to take the scaling limit associated with the CFT: we simply send the gauge coupling $g \rightarrow \infty$. The subtle RG renormalizations of couplings required for the non-supersymmetric case are not required here. Indeed, we may view this ultraviolet insensitivity as the main crutch that is provided by supersymmetry; the infrared physics otherwise remains similar to the non-supersymmetric case.

Finally, the statement of duality is just as in Eqs. (2.2) and (2.3). We define (as in Eq. (12) of KS) the generating function of currents of a dual monopole field $\mathcal{Q}$ by

$$
Z_{\widehat{\mathcal{Q}}}[\widehat{\mathcal{V}}]=\int \mathcal{D} \widehat{\mathcal{Q}} \exp \left(-\int \mathcal{L}_{H}(\widehat{\mathcal{Q}}, \widehat{\mathcal{V}})\right)
$$

and then we have one of the main results of KS

$$
Z_{\mathrm{SQED}-1}[\widehat{\mathcal{V}}]=Z_{\widehat{Q}}[\widehat{\mathcal{V}}]
$$

Here, the universal scaling limits of the two sides are taken simply by the limit $g \rightarrow \infty$. Unlike the non-supersymmetric case, the integrals over the hypermultiplets in $Z_{\text {SQED-1 }}[\widehat{\mathcal{V}}]$ and $Z_{\widehat{o}}[\widehat{\mathcal{V}}]$ are Gaussian, and so can be expressed as superdeterminants-this leads to the key identity in Eq. (13) of KS. We will present a detailed illustration of the result (3.3) in Section 7.1.

\section{4. $N_{f}>1$}

Now we briefly introduce the dualities for $N_{f}>1$. Further discussion of the supersymmetric dualities appears in the sections below.

For the non-supersymmetric case, statements of dualities are only known for an 'easy-plane' extension of $\mathcal{L}_{H}[3,29]$. In this case, we add an additional quartic potential to the Lagrangian, e.g. $\sum_{i}\left|q_{i}\right|^{4}$, so that the continuous global symmetry $S U\left(N_{f}\right)$ symmetry is reduced to $U(1)^{N_{f}-1}$, along with additional discrete symmetries. The dual theory has $N_{f}$ scalar fields $\hat{q}_{i}$ and $N_{f}-1 U(1)$ gauge fields, such that $\hat{q}_{i}$ has charge +1 under the $i$ th $U(1)$ gauge field and charge -1 under the $(i-1)^{\text {th }} U(1)$ gauge field (this is a quiver gauge theory). The identity Eq. (2.3) has a straightforward generalization to this case.

Note that the particle content of the dual theory is identical to that of the direct theory only for $N_{f}=2$, and it was therefore conjectured that the CFT of the Higgs-Coulomb transition is self-dual $[3,30]$.

These dualities generalize to $\mathcal{N}=4$ supersymmetry, as reviewed by KS. However, now the dualities apply also when there is full $S U\left(N_{f}\right)$ flavor symmetry. A theory of $N_{f}$ hypermultiplets $\mathcal{Q}_{i}$ coupled to a vector multiplet $\mathcal{V}$ (this is the theory SQED- $N_{f}$ ) is dual to a theory of $N_{f}$ hypermultiplets $\widehat{\mathcal{Q}}_{i}$ coupled to $N_{f}-1$ vector multiplets, as proven in Section 2.C of KS. These results for $\mathcal{N}=4$ supersymmetry were initially obtained by Intrilligator and Seiberg [15], and are described in their Sections 3.1 and 3.2 for $N_{f}=2$ and $N_{f}>2$ respectively. In addition to the global $S U\left(N_{f}\right)$ flavor symmetry, these theories have a certain $S U(2)_{L} \times S U(2)_{R}$ symmetry required by $\mathcal{N}=4$ supersymmetry. Moreover, there is a global $\widetilde{U}(1)$ symmetry associated with the $U(1)$ gauge invariance in the direct formulation, just as discussed for $\mathcal{L}_{H}$ in Section 1. So the full symmetry is $S U\left(N_{f}\right) \times \widetilde{U}(1) \times S U(2)_{L} \times S U(2)_{R}$. We will see below that the 
$S U\left(N_{f}\right)$ symmetry is broken (preserved) and the $\widetilde{U}(1)$ symmetry is preserved (broken) in the Higgs (Coulomb) phase, as required for a non-LGW transition.

Note again that the particle contents of the direct and dual theories are identical for $N_{f}=2$. In this case the self-duality of the CFT was established by Intrilligator and Seiberg [15]. They also showed that the global symmetry of the CFT is enhanced to $S U\left(2_{f}\right) \times \widetilde{S U}(2) \times S U(2)_{L} \times S U(2)_{R}$, with the first two $S U(2)$ factors exchanging under duality.

\section{Lagrangian with $\mathcal{N}=4$ supersymmetry}

This section will review the matter content of the $\mathcal{N}=4$ multiplets, and the full form of the Lagrangian $\mathcal{L}_{S}$.

First, let us reduce to $\mathcal{N}=2$ superfields. Each $\mathcal{N}=4$ hypermultiplet $\mathcal{Q}_{i}$ consists of a pair of $\mathcal{N}=2$ chiral superfields $\mathcal{Q}_{i}=\left(Q_{i}, \widetilde{Q}^{i}\right)$. Each $\mathcal{N}=4$ vector multiplet, $\mathcal{V}$, consists of a $\mathcal{N}=2$ vector superfield $V$ and a $\mathcal{N}=2$ chiral superfield $\Phi$. The Euclidean Lagrangian in $\mathcal{N}=2$ superspace (without the so-called Fayet-Illiopoulos (FI) term or masses) is

$$
\mathcal{L}_{S}\left(\mathcal{Q}_{i}, \mathcal{V}\right)=\int d^{4} \theta\left(\frac{1}{4 g^{2}} \Sigma^{2}+\frac{1}{g^{2}} \bar{\Phi} \Phi+\bar{Q}_{i} e^{V} Q_{i}+\overline{\widetilde{Q}}^{i} e^{-V} \widetilde{Q}^{i}\right)+\int d^{2} \theta \Phi Q_{i} \widetilde{Q}^{i}+\text { c.c. }
$$

where $i=1 \ldots N_{f}, \theta$ are superspace co-ordinates, $\Sigma=\epsilon^{\alpha \beta} D_{\alpha} \bar{D}_{\beta} V$, and $D_{\alpha}$ are superderivatives. See Appendix A of Ref. [31] for a review of superspace notation in $D=3$.

Turning finally to the explicit components, $V$ consists of $A_{\mu}$, a scalar $\sigma$, the auxiliary field $D$ (which is integrated out), and gaugino $\lambda . \Phi$ consists of a complex scalar $\phi$ and its Fermionic partner $\psi$, as well as the auxiliary field $F .\left(Q_{i}, \widetilde{Q}^{i}\right)$ have components $\left(q_{i}, \tilde{q}^{i}\right)$ complex scalars, $\left(\psi_{q_{i}}, \psi_{\tilde{q}^{i}}\right)$ Dirac Fermions, and auxiliary fields $\left(F_{q_{i}}, F_{\tilde{q}^{i}}\right)$. The full spacetime Lagrangian is then

$$
\begin{aligned}
\mathcal{L}_{S}= & \frac{1}{g^{2}}\left[\frac{1}{4} F_{\mu \nu}^{2}+\frac{1}{2}\left(\partial_{\mu} \sigma\right)^{2}+\frac{1}{2} D^{2}+\bar{\lambda} \sigma^{\mu} \partial_{\mu} \lambda+\left|\partial_{\mu} \phi\right|^{2}+|F|^{2}+\bar{\psi} \sigma^{\mu} \partial_{\mu} \psi\right]+\left|\nabla_{\mu} q_{i}\right|^{2}+\left|\nabla_{\mu} \tilde{q}^{i}\right|^{2} \\
& +\left|\sigma q_{i}\right|^{2}+\left|\sigma \tilde{q}^{i}\right|^{2}+D\left(\left|q_{i}\right|^{2}-\left|\tilde{q}_{i}\right|^{2}\right)+\left|F_{q_{i}}\right|^{2}+\left|F_{\tilde{q}^{i}}\right|^{2}+\bar{\psi}_{q_{i}} \sigma^{\mu} \nabla_{\mu} \psi_{q_{i}}+\bar{\psi}_{\tilde{q}^{i}} \sigma^{\mu} \nabla_{\mu} \psi_{\tilde{q}^{i}} \\
& +\bar{\psi}_{q_{i}} \sigma \psi_{q_{i}}-\bar{\psi}_{\tilde{q}^{i}} \sigma \psi_{\tilde{q}^{i}}+i \bar{q}_{i} \bar{\lambda} \psi_{\tilde{q}^{i}}-i \bar{\psi} \overline{\tilde{q}}_{\tilde{q}^{i}} \lambda q_{i}-i \overline{\tilde{q}}_{i} \bar{\lambda} \psi_{q_{i}}+i \bar{\psi} \bar{q}_{q_{i}} \lambda \tilde{q}^{i} \\
& +\left(F q_{i} \tilde{q}^{i}+\phi F_{q_{i}} \tilde{q}^{i}+\phi q_{i} F_{\tilde{q}^{i}}+\phi \psi_{q_{i}} \psi_{\tilde{q}^{i}}+\psi q_{i} \psi_{\tilde{q}^{i}}+\psi \psi_{q_{i}} \tilde{q}^{i}+\text { c.c. }\right)
\end{aligned}
$$

where $\nabla_{\mu} \equiv \partial_{\mu}-i A_{\mu}$. Integrating out $D, F$, and $F_{q_{i}}, F_{\tilde{q}^{i}}$ yields the potential terms

$$
V=\frac{1}{2}\left(\sum_{i}\left|q_{i}\right|^{2}-\sum_{i}\left|\tilde{q}^{i}\right|^{2}\right)^{2}+\left|\sum_{i} q_{i} \tilde{q}_{i}\right|^{2}+\sum_{i}\left(\left|\phi q_{i}\right|^{2}+\left|\phi \tilde{q}^{i}\right|^{2}\right)
$$

Let us write

$$
q_{i A}=\left(q_{i},-\overline{\tilde{q}}^{i}\right), \quad \psi_{i a}=\left(\psi_{q_{i}},-\bar{\psi}_{\tilde{q}^{i}}\right)
$$

where $a, A=1,2$ are $S U(2)_{L} \times S U(2)_{R}$ spinor indices.

We will denote the $S U(2)_{L}$ triplet $\left(\sigma, \phi=\phi_{1}+i \phi_{2}\right)$ by the real symmetric field $\phi^{(a b)}$. The gauginos $(\lambda, \psi)$ transform as $(\mathbf{2}, \mathbf{2})$ under $S U(2)_{L} \times S U(2)_{R}$, and will be denoted by $\chi_{a A}$. They satisfy the reality condition $\left(\chi_{a A}\right)^{\dagger}=\chi^{a A}=\epsilon^{a b} \epsilon^{A B} \chi_{b B}$.

Now the total scalar potential term can be written as

$$
V=\frac{1}{2} \sum_{I=1}^{3}\left[\bar{q}_{i}^{A}\left(\sigma^{I}\right)_{A}^{B} q_{i B}\right]^{2}+\left|\phi^{(a b)} q_{i A}\right|^{2}
$$

The Fermion-Boson coupling can be written as

$$
\bar{\psi}_{i a} \phi^{(a b)} \psi_{i b}+q_{i A} \chi^{a A} \psi_{i a}+\bar{q}_{i A} \bar{\chi}^{a A} \bar{\psi}_{i a}
$$


Finally, the full Lagrangian in manifestly $S U(2)_{L} \times S U(2)_{R} \times S U\left(N_{f}\right)$ invariant notation is

$$
\begin{aligned}
\mathcal{L}_{S}= & \frac{1}{g^{2}}\left[\frac{1}{4} F_{\mu \nu}^{2}+\left|\partial_{\mu} \phi^{(a b)}\right|^{2}+\chi^{a A} \sigma^{\mu} \partial_{\mu} \chi_{a A}\right]+\left|\nabla_{\mu} q_{i A}\right|^{2}+\frac{1}{2} \sum_{I}\left[\bar{q}_{i}^{A}\left(\sigma^{I}\right)_{A}^{B} q_{i B}\right]^{2}+\left|\phi^{(a b)} q_{i A}\right|^{2} \\
& +\bar{\psi}_{i}^{a} \sigma^{\mu} \nabla_{\mu} \psi_{i a}+\bar{\psi}_{i a} \phi^{(a b)} \psi_{i b}+q_{i A} \chi^{a A} \psi_{i a}+\bar{q}_{i A} \bar{\chi}^{a A} \bar{\psi}_{i a} .
\end{aligned}
$$

The complete field content, along with their transfromations under $S U(2)_{L} \times S U(2)_{R} \times S U\left(N_{f}\right)$, is

- the gauge field $A_{\mu}$, transforming as $(\mathbf{1}, \mathbf{1}, 1)$ (as we will see in Section 7 it is convenient to represent this by a dual scalar $\Sigma$, and the monopole operator $\hat{q} \sim e^{2 \pi i \Sigma}$ ),

- the matter complex scalars $q_{i A}$, transforming as $\left(\mathbf{1}, \mathbf{2}, N_{f}\right)$,

- the matter two-component Dirac Fermions $\psi_{i a}$, transforming as $\left(\mathbf{2}, \mathbf{1}, N_{f}\right)$,

- the gauginos (also two-component Dirac Fermions) $\chi_{a A}$, transforming as $(\mathbf{2}, \mathbf{2}, 1)$ ), and

- the real scalars $\phi^{(a b)}$, transforming as $(\mathbf{3}, \mathbf{1}, 1)$.

The first three fields above, are direct analogs of the fields present in theories of deconfined critical points in doped antiferromagnets as described by Eq. (1.4).

\section{Supersymmetric phase diagram}

The exact phase diagram (moduli space) for the $\mathcal{N}=4$ theory is obtained by minimizing the effective potential in Eq. (5.3) or (5.5)-this potential is protected by supersymmetry. Note that the minimum energy is always $V=0$, and this equation then specifies the structure of the moduli space. The low energy excitations above the vacuum are described by a "sigma model" on this moduli space, and the gradient terms of this sigma model specify the "metric" on moduli space. Because of the special nature of the effective potential (5.5), the moduli space is not simply determined by the structure of the broken symmetry (as it is in non-supersymmetric sigma models). Rather, the target space (moduli space) is a measure of the space along which the effective potential remains flat, and this has additional constraints imposed by supersymmetry.

For $N_{f}>1$, the solution of $V=0$ defines a space with two distinct branches, which will be the Coulomb and Higgs branches. These meet at a singular point (the "origin"), which specifies a CFT separating these branches. For $N_{f}=1$, there is only branch, which will be identified as the Coulomb branch, and so no phase transition. Nevertheless, as we will see below in Section 7, there is a dual representation of the theory on this branch which matches closely with the Dasgupta-Halperin duality of the Abelian Higgs model, as already indicated in Eq. (3.3).

The "Coulomb" branch of the theory has $\phi^{(a b)} \neq 0$ and so we must have $q_{i}=\tilde{q}^{i}=0$ to reach $V=0$. This branch breaks the dual symmetry $\widetilde{U}(1)$, and also $S U(2)_{L}$, while $S U\left(N_{f}\right)$ and $S U(2)_{R}$ are preserved. It is easily seen that the matter fields are massive in fluctuations about any point on this branch. As will be explained in Section 7, classically, the Coulomb branch moduli space is $\mathbb{R}^{3} \times S^{1}$, parameterized by $\phi^{(a b)}$ together with the dual photon $\Sigma$. We will explore the structure of the quantum fluctuation corrections to the moduli space in Section 7: the Coulomb branch moduli space is deformed to a TaubNUT space [32] of NUT charge $N_{f}$, in which the circle is non-trivially fibered over the $\mathbb{R}^{3}$. On any sphere surrounding the origin of the $\mathbb{R}^{3}$, the circle bundle over the sphere has degree $N_{f}$, and its total space is $S^{3} / \mathbb{Z}_{N_{f}}$. Near the origin, the quantum corrected Coulomb branch moduli space looks like $\mathbb{R}^{4} / \mathbb{Z}_{N_{f}}$ rather than $\mathbb{R}^{3} \times S^{1}$.

The "Higgs" branch has $q_{i A} \neq 0$ and $\phi^{a b}=0$, and is present only for $N_{f}>1$. Now the $S U\left(N_{f}\right)$ and $S U(2)_{R}$ symmetries are broken, while $\widetilde{U}(1)$ and $S U(2)_{R}$ are preserved. Comparing with the broken symmetries in the Coulomb branch, we observe that the broken and preserved symmetries are exactly interchanged, and so a direct transition between them is a non-LGW transition. The moduli space of the Higgs branch will be described in Section 8; in this case a classical (i.e. tree-level) analysis of $\mathcal{L}_{S}$ yields the correct structure, and quantum corrections are not as important as in the Coulomb branch. 
The two branches meet at the CFT at the origin of the moduli space $\phi^{(a b)}=0$ and $q_{i A}=0$. For $N_{f}=2$, we will see in Sections 7 and 8 that the moduli metrics of the Coulomb and Higgs branches are identical to each other near the origin, demonstrating the self-duality of this case.

\section{The Coulomb branch}

Because $\phi^{(a b)} \neq 0$, the matter fields $q_{i A}$ and $\psi_{i a}$ are massive. So let us integrate these fields out examine the structure of the effective action at low momenta. The discussion below is an elaborated version of arguments by Seiberg and Witten [14].

We first examine the terms induced in the effective action for the gapless bosonic modes on moduli space: these are the real scalars $\phi^{(a b)}$, and the gauge field $A_{\mu}$. It is convenient to write the scalars in vector notation as $\phi^{\alpha} \tau_{\alpha}^{(a b)}$, where $\tau_{\alpha}$ are the Pauli matrices with $\alpha=x, y, z$. Then the induced effective action for the scalars and the gauge field $A_{\mu}$ is

$$
\delta S_{\phi, A}=N_{f} \operatorname{Tr}_{A} \ln \left[-\nabla_{\mu}^{2}+\phi^{\alpha 2}\right]-N_{f} \operatorname{Tr}_{a} \ln \left[\sigma^{\mu} \nabla_{\mu}+\phi^{\alpha} \tau_{\alpha}\right]
$$

For constant $\phi^{\alpha}$, independent of spacetime co-ordinate $x$, it is now evident that $\delta S_{\phi, A}=0$, because the spectra of the two operators co-incide. Therefore, there is no renormalization of the $\phi^{\alpha}$ superpotential.

To allow for $x$ dependence, we write

$$
\phi^{\alpha}(x)=|\phi| n^{\alpha}+\delta \phi^{\alpha}(x)
$$

where $|\phi|$ is a constant, $n^{\alpha}$ is a constant unit vector, and $\delta \phi^{\alpha}(x)$ is a spacetime dependent fluctuation. Now we can expand in powers of $\delta \phi^{\alpha}$ and $A_{\mu}$. The lowest order terms are of the form

$$
\delta S_{\phi, A}=N_{f} \int \frac{d^{3} p}{8 \pi^{3}}\left[\frac{1}{2} K_{\alpha \beta}(p) \delta \phi^{\alpha}(-p) \delta \phi^{\beta}(p)+\frac{1}{2} G_{\mu v}(p) A_{\mu}(-p) A_{v}(p)+\cdots\right]
$$

The linear coupling between $\delta \phi_{\alpha}$ and $A_{\mu}$ is easily shown to vanish. We now list the expressions for the kernels, initially writing down the contributions of the Bosonic and Fermionic loops separately

$$
\begin{aligned}
K_{\alpha \beta}(p)= & \int \frac{d^{3} q}{8 \pi^{3}}\left[\frac{-8 n_{\alpha} n_{\beta}|\phi|^{2}}{\left(q^{2}+|\phi|^{2}\right)\left((q+p)^{2}+|\phi|^{2}\right)}+\frac{4 \delta_{\alpha \beta}}{\left(q^{2}+|\phi|^{2}\right)}\right] \\
& +\int \frac{d^{3} q}{8 \pi^{3}}\left[\frac{-4 q \cdot(q+p) \delta_{\alpha \beta}+4|\phi|^{2}\left(2 n_{\alpha} n_{\beta}-\delta_{\alpha \beta}\right)}{\left(q^{2}+|\phi|^{2}\right)\left((q+p)^{2}+|\phi|^{2}\right)}\right] \\
= & 2 p^{2} \delta_{\alpha \beta} \int \frac{d^{3} q}{8 \pi^{3}} \frac{1}{\left((q+p / 2)^{2}+|\phi|^{2}\right)\left((q-p / 2)^{2}+|\phi|^{2}\right)}=\delta_{\alpha \beta} \frac{p^{2}}{4 \pi|\phi|} \quad \text { for }|p| \ll|\phi|
\end{aligned}
$$

Note that $K_{\alpha \beta}(0)=0$, as expected from the vanishing of the renormalization of the superpotential. For the kernel of the gauge field, we obtain

$$
\begin{aligned}
G_{\mu v}(p)= & \int \frac{d^{3} q}{8 \pi^{3}}\left[\frac{-2\left(2 q_{\mu}+p_{\mu}\right)\left(2 q_{v}+p_{v}\right)}{\left(q^{2}+|\phi|^{2}\right)\left((q+p)^{2}+|\phi|^{2}\right)}+\frac{4 \delta_{\mu v}}{\left(q^{2}+|\phi|^{2}\right)}\right] \\
& +4 \int \frac{d^{3} q}{8 \pi^{3}}\left[\frac{2 q_{\mu} q_{v}+p_{\mu} q_{v}+p_{v} q_{\mu}-\delta_{\mu v}\left(q \cdot(q+p)+|\phi|^{2}\right)}{\left(q^{2}+|\phi|^{2}\right)\left((q+p)^{2}+|\phi|^{2}\right)}\right] \\
= & \frac{\left(p^{2} \delta_{\mu v}-p_{\mu} p_{v}\right)}{12 \pi|\phi|}+\frac{\left(p^{2} \delta_{\mu v}-p_{\mu} p_{v}\right)}{6 \pi|\phi|} \text { for }|p| \ll|\phi|=\frac{\left(p^{2} \delta_{\mu v}-p_{\mu} p_{v}\right)}{4 \pi|\phi|} \text { for }|p| \ll|\phi|
\end{aligned}
$$

At higher order, there is a triangle diagram which leads to a cubic term between the $A_{\mu}$ and $\delta \phi^{\alpha}$. This arises only from the Fermion loop and leads to a contribution of the form 


$$
\begin{gathered}
A_{\mu}\left(-p_{1}-p_{2}\right) \delta \phi^{\alpha}\left(p_{1}\right) \delta \phi^{\beta}\left(p_{2}\right) \times\left[4 i \epsilon_{\mu \nu \lambda} p_{1 v} p_{2 \lambda} \epsilon_{\alpha \beta \gamma} n^{\gamma}|\phi| \int \frac{d^{3} q}{8 \pi^{3}} \frac{1}{\left(q^{2}+|\phi|^{2}\right)^{3}}\right] \\
=\frac{i}{8 \pi|\phi|^{2}} \epsilon_{\mu \nu \lambda} p_{1 v} p_{2 \lambda} \epsilon_{\alpha \beta \gamma} n^{\gamma} A_{\mu}\left(-p_{1}-p_{2}\right) \delta \phi^{\alpha}\left(p_{1}\right) \delta \phi^{\beta}\left(p_{2}\right)
\end{gathered}
$$

Putting all these terms together, we have the effective action in the Coulomb branch

$$
\begin{aligned}
S_{\phi, A} & =\int d^{3} x\left[\frac{1}{2 \widetilde{g}^{2}}\left[\left(\partial_{\mu} \phi^{\alpha}\right)^{2}+\left(\epsilon_{\mu \nu \lambda} \partial_{\nu} A_{\lambda}\right)^{2}\right]+\frac{i N_{f}}{8 \pi|\phi|^{3}} \epsilon_{\mu \nu \lambda} \epsilon_{\alpha \beta \gamma} A_{\mu} \phi^{\alpha} \partial_{\nu} \phi^{\beta} \partial_{\lambda} \phi^{\gamma}\right] \\
& =\int d^{3} x\left[\frac{1}{2 \tilde{g}^{2}}\left(\left(\partial_{\mu} \phi^{\alpha}\right)^{2}+\left(\widetilde{F}^{\mu}\right)^{2}\right)+\frac{i N_{f}}{4 \pi} \widetilde{F}^{\mu} \partial_{\mu} \phi^{\alpha} \mathcal{A}_{\alpha}\right]
\end{aligned}
$$

where the renormalized coupling is

$$
\frac{1}{\widetilde{g}^{2}(x)}=\frac{1}{g^{2}}+\frac{N_{f}}{4 \pi \sqrt{\phi^{\alpha}(x) \phi^{\alpha}(x)}}
$$

and $\widetilde{F}^{\mu} \equiv \epsilon^{\mu v \rho} \partial_{n u} A_{\rho}$. Here, and henceforth, we will consider the coupling $\widetilde{g}$ and $|\phi|=\sqrt{\phi^{\alpha}(x) \phi^{\alpha}(x)}$ to be arbitrary functions of the spacetime co-ordinate $x$. We have also introduced the Dirac monopole function $\mathcal{A}_{\alpha}$ on $\phi^{\alpha}$ space which obeys

$$
\frac{\partial \mathcal{A}_{\alpha}}{\partial \phi^{\beta}}-\frac{\partial \mathcal{A}_{\beta}}{\partial \phi^{\alpha}}=\frac{\epsilon_{\alpha \beta \gamma} \phi^{\gamma}}{|\phi|^{3}}
$$

Then we can verify that

$$
\epsilon_{\mu \nu \lambda} \partial_{\nu}\left[\mathcal{A}_{\alpha} \partial_{\lambda} \phi^{\alpha}\right]=\frac{1}{2|\phi|^{3}} \epsilon_{\mu \nu \lambda} \epsilon_{\alpha \beta \gamma} \phi^{\alpha} \partial_{\nu} \phi^{\beta} \partial_{\lambda} \phi^{\gamma}
$$

Eq. (7.7) defines the bosonic sector of the sigma model on the Coulomb branch of moduli space, expressed in terms of the real scalar $\phi^{\alpha}$ and the gauge field $A_{\mu}$. We can also use similar techniques to obtain the Fermionic sector, which would be an effective action for $\chi_{a A}$. Rather than working this out from the Feynman graph expansion, we choose to determined the Fermion Lagrangian from the bosonic sector by supersymmetry. It is of the form

$$
S_{F}=\int d^{3} x\left\{\frac{1}{2 \tilde{g}^{2}} \chi \sigma^{\mu} \partial_{\mu} \chi+\frac{1}{2} \chi \sigma^{\mu} \tau^{\alpha} \mathcal{V}_{\mu \alpha}(\phi, \widetilde{F}) \chi+\mathcal{O}\left(\chi^{4}\right)\right\}
$$

where the contraction of spinor and $S U(2) \times S U(2)$ indices are understood. The supersymmetry transformations are

$$
\begin{aligned}
& \delta \phi^{\alpha}=\varepsilon^{a A}\left(\tau^{\alpha}\right)_{a}^{b} \chi_{b A}=\varepsilon \tau^{\alpha} \chi \\
& \delta A_{\mu}=i \varepsilon^{a A} \sigma_{\mu} \chi_{a A}=i \varepsilon \sigma_{\mu} \chi \\
& \delta \chi_{a A}=\left[-\left(\tau_{\alpha}\right)_{a}^{b} \sigma^{\mu} \partial_{\mu} \phi^{\alpha}+\delta_{a}^{b} \sigma^{\mu} \widetilde{F}_{\mu}\right] \varepsilon_{b A}=\left[\left(-\tau_{\alpha} \sigma^{\mu} \partial_{\mu} \phi^{\alpha}+\sigma^{\mu} \widetilde{F}_{\mu}\right) \varepsilon\right]_{a A} .
\end{aligned}
$$

The supersymmetry variation of the bosonic part of the action is

$$
\begin{aligned}
\delta S_{\phi, A}= & \int d^{3} \chi\left\{\left(\frac{1}{\tilde{g}^{2}} \partial^{\mu} \phi_{\alpha}+\frac{i N_{f}}{4 \pi} \mathcal{A}_{\alpha} \widetilde{F}^{\mu}\right) \varepsilon \tau^{\alpha} \partial_{\mu} \chi+\left[-\frac{\partial_{\alpha} \tilde{g}}{\tilde{g}^{3}}\left((\partial \phi)^{2}+\widetilde{F}^{2}\right)+\frac{i N_{f}}{4 \pi} \partial_{\alpha} \mathcal{A}_{\beta} \widetilde{F}^{\mu} \partial_{\mu} \phi^{\beta}\right] \varepsilon \tau^{\alpha} \chi\right. \\
& \left.-i \epsilon^{\mu \nu \rho}\left(\frac{1}{\tilde{g}^{2}} \widetilde{F}_{\rho}+\frac{i N_{f}}{4 \pi} \mathcal{A}_{\alpha} \partial_{\rho} \phi^{\alpha}\right) \varepsilon \sigma_{\mu} \partial_{v} \chi\right\}=-\int d^{3} x\left\{\chi \tau ^ { \alpha } \varepsilon \left[-\partial_{\mu}\left(\frac{1}{\tilde{g}^{2}} \partial^{\mu} \phi_{\alpha}+\frac{i N_{f}}{4 \pi} \mathcal{A}_{\alpha} \widetilde{F}^{\mu}\right)\right.\right. \\
& \left.\left.-\frac{\partial_{\alpha} \tilde{g}}{\tilde{g}^{3}}\left((\partial \phi)^{2}+\widetilde{F}^{2}\right)+\frac{i N_{f}}{4 \pi} \partial_{\alpha} \mathcal{A}_{\beta} \widetilde{F}^{\mu} \partial_{\mu} \phi^{\beta}\right]+\chi \sigma_{\mu} \varepsilon i \epsilon^{\mu \nu \rho} \partial_{v}\left(\frac{1}{\tilde{g}^{2}} \widetilde{F}_{\rho}+\frac{i N_{f}}{4 \pi} \mathcal{A}_{\alpha} \partial_{\rho} \phi^{\alpha}\right)\right\}
\end{aligned}
$$


The supersymmetry variation of the Fermion action is

$$
\begin{aligned}
\delta S_{F}= & \int d^{3} \chi\left\{\frac{1}{2 \tilde{g}^{2}} \chi \sigma^{\mu} \partial_{\mu}\left(-\tau_{\alpha} \sigma^{v} \partial_{v} \phi^{\alpha}+\sigma^{\nu} \widetilde{F}_{v}\right) \varepsilon+\frac{1}{2} \chi \sigma^{\mu} \partial_{\mu}\left[\frac{1}{\tilde{g}^{2}}\left(-\tau_{\alpha} \sigma^{v} \partial_{v} \phi^{\alpha}+\sigma^{\nu} \widetilde{F}_{v}\right)\right] \varepsilon\right. \\
& \left.+\mathcal{V}_{\mu \alpha}(\phi, \widetilde{F}) \chi \sigma^{\mu} \tau^{\alpha}\left(-\tau_{\beta} \sigma^{v} \partial_{v} \phi^{\beta}+\sigma^{\nu} \widetilde{F}_{v}\right) \varepsilon+\mathcal{O}\left(\chi^{3}\right)\right\}
\end{aligned}
$$

By the canceling the term proportional to $\chi \varepsilon$ and $\chi \sigma_{\mu} \tau_{\alpha} \varepsilon$, we find

$$
\mathcal{V}_{\mu \alpha}=\frac{1}{2} \partial_{\alpha}\left(\frac{1}{\tilde{g}^{2}}\right) \widetilde{F}_{\mu}-\frac{i}{2} \epsilon_{\alpha \beta \gamma} \partial_{\beta}\left(\frac{1}{\tilde{g}^{2}}\right) \partial_{\mu} \phi^{\gamma}=\frac{N_{f}}{8 \pi|\phi|^{3}}\left(-\phi^{\alpha} \widetilde{F}_{\mu}+i \epsilon_{\alpha \beta \gamma} \phi^{\beta} \partial_{\mu} \phi^{\gamma}\right)
$$

One can check that the terms proportional to $\chi \sigma_{\mu} \varepsilon$ and $\chi \tau_{\alpha} \varepsilon$ cancel the variation of the bosonic part of the action.

In the $g \rightarrow \infty$ limit, we can write the Fermion action as

$$
S_{F, g \rightarrow \infty}=\frac{N_{f}}{8 \pi} \int d^{3} \chi\left[\frac{1}{|\phi|} \chi \sigma^{\mu} \partial_{\mu} \chi+\frac{1}{2|\phi|^{3}} \chi\left(-\phi^{\alpha} \tau_{\alpha} \sigma^{\mu} \widetilde{F}_{\mu}+\phi^{\alpha} \tau_{\alpha} \sigma^{\mu} \partial_{\mu} \phi^{\beta} \tau_{\beta}\right) \chi+\mathcal{O}\left(\chi^{4}\right)\right]
$$

In the remainder of this section, we will rewrite the sigma model in Eqs. (7.7) and (7.16) in a different set of 'vortex' variables, designed to highlight the duality properties. Decoupling the gauge field kinetic energy by a Hubbard-Stratonovich field $\widehat{A}_{\mu}$ in Eq. (7.7), we obtain

$$
S_{\phi, A}=\int d^{3} x\left[\frac{1}{2 \widetilde{g}^{2}}\left(\partial_{\mu} \phi^{\alpha}\right)^{2}+\frac{\widetilde{g}^{2}}{2} \widehat{A}_{\mu}^{2}+i A_{\mu} \epsilon_{\mu \nu \lambda} \partial_{\nu}\left(K_{\lambda}+\frac{N_{f}}{4 \pi} \mathcal{A}_{\alpha} \partial_{\lambda} \phi^{\alpha}\right)\right]
$$

Together with the Fermion Lagrangian, and performing the integral over $A_{\mu}$, we obtain a constraint equation which is solved by a dual field $\Sigma$ to yield the dual action

$$
\begin{aligned}
\widetilde{S}= & \int d^{3} x\left[\frac{1}{2 \widetilde{g}^{2}}\left(\partial_{\mu} \phi^{\alpha}\right)^{2}+\frac{\widetilde{g}^{2}}{2}\left(\partial_{\mu} \Sigma-\frac{N_{f}}{4 \pi} \mathcal{A}_{\alpha} \partial_{\mu} \phi^{\alpha}-\frac{i N_{f} \phi^{\alpha}}{16 \pi|\phi|^{3}} \chi \tau_{\alpha} \sigma_{\mu} \chi\right)^{2}\right. \\
& \left.+\frac{1}{2 \widetilde{g}^{2}} \chi \sigma^{\mu} \partial_{\mu} \chi+\frac{i N_{f}}{16 \pi} \epsilon_{\alpha \beta \gamma} \frac{\phi^{\alpha}}{|\phi|^{3}}\left(\partial_{\mu} \phi^{\beta}\right) \chi \tau^{\gamma} \sigma^{\mu} \chi+\mathcal{O}\left(\chi^{4}\right)\right]
\end{aligned}
$$

This is the new form of our sigma model on the Coulomb branch, now expressed in terms of the real scalar $\phi^{\alpha}$, a new scalar $\Sigma$, and the Dirac Fermions $\chi$. Thus we have exchanged the photon $A_{\mu}$ for a dual scalar $\Sigma$, and from the arguments in Section 1 we can anticipate that $e^{2 \pi i \Sigma}$ is the monopole operator. We will see this emerge in the analyses below.

\subsection{Duality for $N_{f}=1$}

We have now assembled the ingredients to illustrate the origin of the key duality relation in Eq. (3.3) for the $\mathcal{N}=4$ theory for $N_{f}=1$.

The crucial and remarkable point is that in the limit where we can take $\widetilde{g}^{2}=4 \pi|\phi|$, Eq. (7.18) is actually a free field theory. To see this, it is useful to introduce spherical polar co-ordinates $(\rho, \theta, \gamma)$ in $\phi^{\alpha}$ space so that $\phi^{\alpha}=\rho(\sin \theta \cos \gamma, \sin \theta \sin \gamma, \cos \theta)$, where $\rho, \theta$, and $\gamma$ are functions of $x$. Also, let us choose

$$
\mathcal{A}_{\alpha}=\frac{\sin \theta}{\rho(1+\cos \theta)}(\sin \gamma,-\cos \gamma, 0)
$$

so that Eq. (7.9) is obeyed. Then, we define, the two component complex field $\hat{q}_{a}$ by

$$
\hat{q}_{a}=\sqrt{\frac{\rho}{2 \pi}} e^{2 \pi i \Sigma}\left(\begin{array}{c}
\cos (\theta / 2) \\
\sin (\theta / 2) e^{i \gamma}
\end{array}\right)
$$


From this parameterization, we see that $\Sigma$ lives on a circle with circumference 1 ; This is consistent with Eq. (7.18) where the line integral of $\mathcal{A}_{\alpha} \partial_{\mu} \phi^{\alpha}$ is defined modulo the area of the unit sphere, which is $4 \pi$. The field $\hat{q}_{a}$ is clearly the bosonic monopole operator. An explicit computation shows easily that the bosonic part of the action $\widetilde{S}$ in Eq. (7.18) is the free field theory $S_{\hat{q}}$ for $N_{f}=1$ and $\tilde{g}^{2}=4 \pi|\phi|$, where

$$
S_{\hat{q}}=\int d^{3} x\left|\partial_{\mu} \hat{q}_{a}\right|^{2}
$$

Note that under $S U(2)_{L} \times S U(2)_{R}, \hat{q}_{a}$ transforms as a $(\mathbf{2}, 1)$. The $\hat{q}_{a}$ also carry a charge under a global $\widetilde{U}(1)_{f}$ symmetry which is the dual of the $U(1)$ gauge field. Under this $\widetilde{U}(1)_{f}$ symmetry $\Sigma \rightarrow \Sigma+c$, where $c$ is a constant.

A similar analysis can be carried out for the Fermionic fields. These consist of $\chi_{a A}$ gauginos which transform under $S U(2)_{L} \times S U(2)_{R}$ as a $(\mathbf{2}, \mathbf{2})$, and obey a reality condition. After integrating out the matter fields, for $N_{f}=1$, these Fermions should be transformed to the superpartners of the $\hat{q}_{a}$ : i.e. they should become a complex doublet, $\hat{\psi}_{A}$ which transforms as a $(1,2)$ and carries a $\widetilde{U}(1)_{f}$ charge, and has a free Dirac Lagrangian. The resulting dual description is therefore simply that of a free $\mathcal{N}=4$ hypermultiplet, $\widehat{\mathcal{Q}}$. This is the content of the duality relation in Eq. (3.3).

In the $g \rightarrow \infty$ limit, the $\mathcal{O}\left(\chi^{4}\right)$ terms must vanish because the target space of the sigma model is locally flat $\mathbb{R}^{4}$ (away from the origin). We can then write the Fermionic terms in the action $\widetilde{S}$ in Eq. (7.18) as

$$
\widetilde{S}_{F, g \rightarrow \infty}=\frac{N_{f}}{8 \pi} \int d^{3} \chi\left[\frac{1}{|\phi|} \chi \sigma^{\mu} \partial_{\mu} \chi+\frac{1}{2|\phi|^{3}} \chi \phi^{\alpha} \tau_{\alpha} \sigma^{\mu} \partial_{\mu} \phi^{\beta} \tau_{\beta} \chi-\frac{i}{2|\phi|^{2}} \chi \phi^{\beta} \tau_{\beta}\left(\frac{4 \pi}{N_{f}} \sigma^{\mu} \partial_{\mu} \Sigma-\mathcal{A}_{\alpha} \sigma^{\mu} \partial_{\mu} \phi^{\alpha}\right) \chi\right]
$$

In terms of

$$
\hat{q}=\sqrt{\frac{\rho}{2 \pi}} e^{2 \pi i \Sigma / N_{f}}\left(\begin{array}{c}
\cos (\theta / 2) \\
\sin (\theta / 2) e^{i \gamma}
\end{array}\right)
$$

we have

$$
\begin{aligned}
& \phi^{\alpha}=2 \pi \hat{q}^{\dagger} \tau^{\alpha} \hat{q},|\phi|=2 \pi|\hat{q}|^{2} \\
& \phi^{\alpha} \tau_{\alpha}=2 \pi\left(2 \hat{q} \hat{q}^{\dagger}-|\hat{q}|^{2}\right), \\
& i|\phi|\left(\frac{4 \pi}{N_{f}} \partial_{\mu} \Sigma-\mathcal{A}_{\alpha} \partial_{\mu} \phi^{\alpha}\right)=\hat{q}^{\dagger} \partial_{\mu} \hat{q}-\partial_{\mu} \hat{q}^{\dagger} \hat{q} .
\end{aligned}
$$

Now we can rewrite (7.22) as

$$
\begin{aligned}
\widetilde{S}_{F, g \rightarrow \infty} & =\frac{N_{f}}{16 \pi^{2}} \int d^{3} x \frac{1}{|\hat{q}|^{4}}\left[|\hat{q}|^{2} \chi \sigma^{\mu} \partial_{\mu} \chi-\left(\chi \partial_{\mu} \hat{q}\right) \sigma^{\mu}\left(\hat{q}^{\dagger} \chi\right)+(\chi \hat{q}) \sigma^{\mu}\left(\partial_{\mu} \hat{q}^{\dagger} \chi\right)\right] \\
& =\frac{N_{f}}{8 \pi^{2}} \int d^{3} x\left(\frac{\chi \hat{q}}{|\hat{q}|^{2}}\right) \sigma^{\mu} \partial_{\mu}\left(\frac{\hat{q}^{\dagger} \chi}{|\hat{q}|^{2}}\right)
\end{aligned}
$$

So we see that $\chi_{a A}$ can be mapped to an $S U(2)_{R}$ doublet of complex Fermions $\psi_{A}$,

$$
\hat{\psi}=\sqrt{\frac{N_{f}}{2}} \hat{q}^{\dagger} \chi 2 \pi|\hat{q}|^{2}
$$

which are free away from the origin of the moduli space $\hat{q}=0$ with action

$$
S_{\hat{\psi}}=\int d^{3} x \overline{\hat{\psi}} \sigma^{\mu} \partial_{\mu} \hat{\psi} .
$$

Thus Eqs. (7.21) and (7.27) show that when $N_{f}=1$, the moduli space is smooth and reduces to flat $\mathbb{R}^{4}$. This establishes the equivalence of SQED- 1 to the theory of a free $\mathcal{N}=4$ hypermultiplet. 


\section{Higgs branch}

We will now consider the sigma model on the Higgs branch of the moduli space. Here we simply have to take the low energy limit of the Lagrangian $\mathcal{L}_{S}$ in Eq. (5.7) about vacuum point where $\phi^{a b}=0$ but $q_{i A} \neq 0$. (recall that there is no such vacuum for $N_{f}=1$ ). The analysis is simpler than on the Coulomb branch, because here we can simply set $\phi^{a b}=0$, and need not include the fluctuation contribution of the massive $\phi^{a b}$ fields. The main analysis needed is to project $\mathcal{L}_{S}$ onto the low energy sector defined by $V=0$.

We will explicit carry out such an analysis for $N_{f}=2$. Our purpose here is to illustrate that the resulting low energy sigma model on the Higgs branch is in fact identical to the dual version of the sigma model obtained on the Coulomb branch in Eq. (7.18). This identity then illustrates the self-duality of the $N_{f}=2$ case, noted by Intrilligator and Seiberg [15].

A point on the Higgs branch for $N_{f}=2$ has $q_{1 A} \neq 0$ and $q_{2 A} \neq 0$. The vanishing of the superpotential implies that

$$
\bar{q}_{1}^{A}\left(\sigma^{I}\right)_{A}^{B} q_{1 B}=-\bar{q}_{2}^{A}\left(\sigma^{I}\right)_{A}^{B} q_{2 B}
$$

for $I=1,2,3$. Let us choose a parameterization of the solutions of this equation which if formally similar to Eq. (7.20).

$$
q_{1 A}=\sqrt{\frac{\rho}{4 \pi}} e^{\pi i \Sigma_{1}}\left(\begin{array}{c}
\cos (\theta / 2) \\
\sin (\theta / 2) e^{i \gamma}
\end{array}\right), \quad q_{2 A}=\sqrt{\frac{\rho}{4 \pi}} e^{\pi i \Sigma_{2}}\left(\begin{array}{c}
\sin (\theta / 2) e^{-i \gamma} \\
-\cos (\theta / 2)
\end{array}\right),
$$

By inserting this parameterization into the Lagrangian $\mathcal{L}_{S}$ in Eq. (5.7), we obtain the action for the bosonic sector of the Higgs branch sigma model. The gauge field $A_{\mu}$ higgses out the combination $\Sigma_{1}+\Sigma_{2}$. So we can set $\Sigma_{1}=-\Sigma_{2} \equiv \Sigma$, and then ignore $A_{\mu}=0$. The resulting action for $\Sigma, \theta$ and $\gamma$ is then found to be identical to the bosonic sector of Eq. (7.18) for $N_{f}=2$, with the parameterization for $\mathcal{A}_{\alpha}$ in Eq. (7.19). Thus, as claimed above, the metric on the moduli spaces of the Higgs and Coulomb branches are identical in the limit $g \rightarrow \infty$. This identity also explains why the symmetry is enhanced from $\widetilde{U}(1)$ to $\widetilde{S U}(2)$ as we approach the singular CFT point on the moduli space.

For general $N_{f}$, the Higgs branch moduli space is a $4\left(N_{f}-1\right)$ real dimensional hyperkähler manifold, which is described as a hyperkähler quotient $\mathbb{C}^{2 N_{f}} U(1)$. The latter is explicitly given by

$$
\begin{aligned}
& \sum_{i=1}^{N_{f}}\left|q_{i}\right|^{2}-\sum_{i=1}^{N_{f}}\left|\tilde{q}^{i}\right|^{2}=\zeta_{\mathbb{R}}, \\
& \sum_{i=1}^{N_{f}} q_{i} \tilde{q}^{i}=\zeta_{\mathbb{C}}
\end{aligned}
$$

and modded out by the $U(1)$ action on $q_{i}$ and $\tilde{q}^{i}$ with charges $(+1,-1)$. Here $\vec{\zeta}=\left(\zeta_{\mathbb{R}}, \zeta_{\mathbb{C}}\right)$ are the $\mathcal{N}=4$ Fayet-Illiopoulos parameters, which we have previously set to zero. In particular, when $N_{f}=2$, the Higgs branch moduli space is known as the $A_{1}$ asymptotically locally Euclidean (ALE) space. For $\vec{\zeta}=0$ it is $\mathbb{C}^{2} / \mathbb{Z}_{2}$, and when $\vec{\zeta} \neq 0$ the $\mathbb{Z}_{2}$ orbifold singularity is replaced (resolved) by a $\mathbb{C P}^{1}$. The Higgs branch moduli space does not receive quantum corrections, and the classical moduli space is exact. This is because the gauge coupling can be promoted to a vector multiplet, while the Higgs branch is parameterized by hypermultiplets. The two decouple at the level of kinetic terms in the low energy effective Lagrangian [34].

\section{Conclusions}

This paper has attempted to straddle the boundaries of two fields, by connecting theories of quantum phase transitions in square lattice antiferromagnets to dualities of supersymmetric field theories in $2+1$ dimensions. We highlighted to the common physical ideas behind duality mappings in these fields, and so found explicit examples of quantum phase transitions which do not obey the LandauGinzburg-Wilson paradiagm. Our analysis was restricted to theories with $\mathcal{N}=4$ supersymmetry, 
where the correspondence was the simplest. Closely related duality mappings are also available with smaller amounts of supersymmetry [33], at the cost of some additional complexity.

We conclude by noting that another recent example of the parallel developments in the theories of two-dimensional antiferromagnets and dualities in supersymmetric gauge theories can be found in the close similarities of the theories and duality mappings described in Refs. [35] and [36].

\section{Acknowledgment}

S.S. would like to thank S. Hartnoll and M. Strassler for very useful discussions. We also thank F. Nogueira for valuable comments on the manuscript. This research was supported by the NSF under grant DMR-0757145. X.Y. was supported by a Junior Fellowship from the Harvard Society of Fellows.

\section{References}

[1] T. Senthil, A. Vishwanath, L. Balents, S. Sachdev, M.P.A. Fisher, Deconfined quantum critical points', Science 303 (2004) 1490. arXiv:cond-mat/0311326.

[2] T. Senthil, L. Balents, S. Sachdev, A. Vishwanath, M.P.A. Fisher, Quantum criticality beyond the Landau-Ginzburg-Wilson paradigm, Phys. Rev. B 70 (2004) 144407. arXiv:cond-mat/0312617.

[3] O.I. Motrunich, A. Vishwanath, Emergent photons and transitions in the $\mathrm{O}(3)$ sigma model with hedgehog suppression, Phys. Rev. B 70 (2004) 075104. arXiv:cond-mat/0311222.

[4] A.W. Sandvik, Evidence for deconfined quantum criticality in a two-dimensional Heisenberg model with four-spin interactions, Phys. Rev. Lett. 98 (2007) 227202. arXiv:0611343.

[5] F.S. Nogueira, S. Kragset, A. Sudbo, Quantum critical scaling behavior of deconfined spinons, Phys. Rev. B 76 (2007) 220403R. arXiv:0708.3633.

[6] R.G. Melko, R.K. Kaul, Scaling in the fan of an unconventional quantum critical point, Phys. Rev. Lett. 100 (2008) 017203. arXiv:0707.2961;

R.K. Kaul, R.G. Melko, Large-N estimates of universal amplitudes of the $C P^{N-1}$ theory and comparison with a $S=\frac{1}{2}$ squarelattice model with competing four-spin interactions, Phys. Rev. B 78 (2008) 014417. arXiv:0804.2279.

[7] F.-J. Jiang, M. Nyfeler, S. Chandrasekharan, U.-J. Wiese, From an antiferromagnet to a valence bond solid: evidence for a first order phase transition (arXiv:0710.3926).

[8] A.B. Kuklov, M. Matsumoto, N.V. Prokof'ev, B.V. Svistunov, M. Troyer, Deconfined criticality: generic first-order transition in the SU(2) symmetry case, Phys. Rev. Lett. 101 (2008) 050405. arXiv:0805.4334.

[9] O.I. Motrunich, A. Vishwanath, Comparative study of Higgs transition in one-component and two-component lattice superconductor models. arXiv:0805.1494

[10] F.D.M. Haldane, Spontaneous dimerization in the $S=1 / 2$ Heisenberg antiferromagnetic chain with competing interactions, Phys. Rev. B 25 (1982) 4925.

[11] N. Read, S. Sachdev, Some features of the phase diagram of SU(N) antiferromagnets on a square lattice, Nucl. Phys. B 316 (1989) 609.

[12] N. Read, S. Sachdev, Valence bond and spin-Peierls ground states of low dimensional quantum antiferromagnets, Phys. Rev. Lett. 62 (1989) 1694.

[13] N. Read, S. Sachdev, Spin-Peierls, valence bond solid, and Neel ground states of low dimensional quantum antiferromagnets, Phys. Rev. B 42 (1990) 4569.

[14] N. Seiberg, E. Witten, Gauge dynamics and compactification to three dimensions. arXiv:hep-th/9607163.

[15] K.A. Intriligator, N. Seiberg, Mirror symmetry in three dimensional gauge theories, Phys. Lett. B 387 (1996) 513. arXiv:hepth/9607207.

[16] A. Kapustin, M.J. Strassler, On mirror symmetry in three dimensional Abelian gauge theories, JHEP 9904 (1999) 021. arXiv:hep-th/9902033.

[17] G. Murthy, S. Sachdev, Action of hedgehog instantons in the disordered phase of the (2+1)-dimensional $C P^{N-1}$ model, Nucl. Phys. B 344 (1990) 557.

[18] A. Kovner, B. Rosenstein, D. Eliezer, Photon as a goldstone boson in (2+1)-dimensional abelian gauge theories, Nucl. Phys. B 350 (1991) 325.

[19] V. Borokhov, A. Kapustin, X.k. Wu, Topological disorder operators in three-dimensional conformal field theory, JHEP 0211 (2002) 049. arXiv:hep-th/0206054.

[20] M.A. Metlitski, S. Sachdev, Valence bond solid order near impurities in two-dimensional quantum antiferromagnets, Phys. Rev. B 77 (2008) 054411. arXiv:0710.0626.

[21] S. Sachdev, Quantum magnetism and criticality, Nature Phys. 4 (2008) 173. arXiv:0711.3015.

[22] S. Sachdev, Quantum phases and phase transitions of Mott insulators, in: U. Schollwock, J. Richter, D.J.J. Farnell, R.A. Bishop (Eds.), Quantum Magnetism, Lecture Notes in Physics, vol. 645, Springer, Berlin, 2004. arXiv:cond-mat/0401041.

[23] F.D.M. Haldane, $O(3)$ Nonlinear $\sigma$ model and the topological distinction between integer- and half-integer-spin antiferromagnets in two dimensions, Phys. Rev. Lett. 61 (1988) 1029.

[24] R.K. Kaul, Y.B. Kim, S. Sachdev, T. Senthil, Algebraic charge liquids, Nature Phys. 4 (2008) 28. arXiv:0706.2187.

[25] F.S. Nogueira, Deconfined quantum criticality driven by Dirac Fermions in SU(2) antiferromagnets, Phys. Rev. B 77 (2008) 195101. arXiv:0802.0500.

[26] R.K. Kaul, S. Sachdev, Quantum criticality of $U(1)$ gauge theories with Fermionic and Bosonic matter in two spatial dimensions, Phys. Rev. B 77 (2008) 155105. arXiv:0801.0723.

[27] M.E. Peskin, Mandelstam-'t Hooft duality in abelian lattice models, Ann. Phys. 113 (1978) 122. 
[28] C. Dasgupta, B.I. Halperin, Phase transition in a lattice model of superconductivity, Phys. Rev. Lett. 47 (1981) 1556.

[29] L. Balents, L. Bartosch, A. Burkov, S. Sachdev, K. Sengupta, Putting competing orders in their place near the Mott transition, Phys. Rev. B 71 (2005) 144508. arXiv:cond-mat/0408329.

[30] C.P. Herzog, P. Kovtun, S. Sachdev, D.T. Son, Quantum critical transport, duality, and M-theory, Phys. Rev. D 75 (2007) 085020. arXiv:hep-th/0701036.

[31] M. Benna, I. Klebanov, T. Klose, M. Smedback, Superconformal Chern-Simons theories and $\mathrm{AdS}_{4} / \mathrm{CFT}_{3}$. Correspondence arXiv:0806.1519.

[32] S.W. Hawking, G.F.R. Ellis, The Large Scale Structure of Spacetime, Cambridge University Press, Cambridge, 1975 (Section 5.8 ).

[33] O. Aharony, A. Hanany, K.A. Intriligator, N. Seiberg, M.J. Strassler, Aspects of $\mathcal{N}=2$ supersymmetric gauge theories in three dimensions, Nucl. Phys. B 499 (1997) 67. hep-th/9703110.

[34] P.C. Argyres, M.R. Plesser, N. Seiberg, The moduli space of $\mathcal{N}=2$ SUSY QCD and duality in $\mathcal{N}=1$ SUSY QCD, Nucl. Phys. B 471 (1996) 159. arXiv:hep-th/9603042.

[35] C. Xu, S. Sachdev, Global phase diagrams of frustrated quantum antiferromagnets in two dimensions: doubled ChernSimons theory, Phys. Rev. B 79 (2009) 064405. arXiv:0811.1220.

[36] D.L. Jafferis, X. Yin, Chern-Simons-Matter theory and mirror symmetry. arXiv:0810.1243. 